\title{
Cystic form of pulmonary sequestration
}

\author{
Sheng-Yuan Ruan MD, Chung-Yi Yang MD, Chong-Jen Yu MD PhD
}

Competing interests: None declared.

This article has been peer reviewed.

Affiliations: From the Departments of Internal Medicine (Ruan) and Medical Imaging (Yang), National Taiwan University Hospital Yun-Lin Branch, Yun-Lin, Taiwan; and the Department of Internal Medicine ( $\mathrm{Yu}$ ), National Taiwan University Hospital, Taipei, Taiwan

Correspondence to:

Dr. Chung-Yi Yang, cyyang@ntu.edu.tw

CMAJ 2011. DOI:10.1503 /cmaj.100203

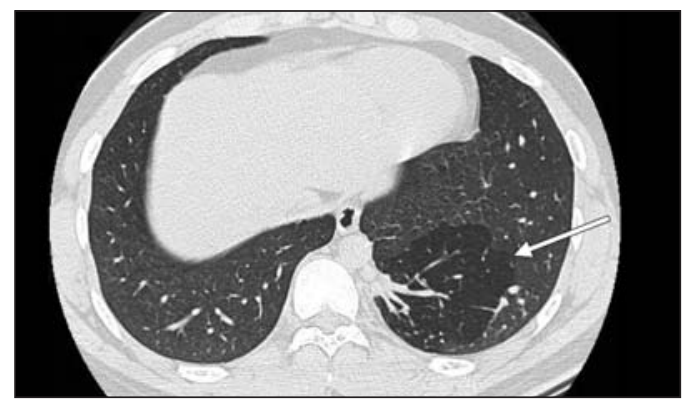

Figure 1: Computed tomographic image of the chest in a 17-year-old boy with hemoptysis showing a well-circumscribed hyperlucent area (arrow) in the left basal lung.

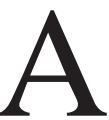

17-year-old boy was referred to our hospital because of repeated episodes of hemoptysis for several years. His physical examination was unremarkable. His blood cell count and coagulation profile were normal. Chest radiography (Appendix 1, available at www.cmaj.ca/cgi/content/full/cmaj.100203/DC1) and bronchoscopy showed no anomalies. Computed tomography (CT) of the chest showed a well-circumscribed hyperlucent area in the left basal lung (Figure 1) with a feeding artery from the aorta and venous return to the azygos vein (Appendix 2, available at www.cmaj.ca/cgi/content /full/cmaj.100203/DC1). The reconstructed CT image showed two feeding arteries from the aorta supplying the left basal lung (Figure 2).

Pulmonary sequestration, the second most common congenital lung anomaly after congenital cystic adenomatoid malformation, refers to a spectrum of abnormal connections of one or more of the four major components: airway, lung parenchyma, arterial supply and venous drainage. ${ }^{1}$ The estimated incidence in referral centres is $0.15 \%-1.8 \% .^{2}$ Pulmonary sequestration is divided into intralobar and extralobar types on the basis of the presence or absence of pleural covering.

Diagnosis requires a high index of suspicion when a child or young adult presents with recurrent lower lobe pneumonia, hemoptysis, shortness of breath or chest pain. ${ }^{2.3}$ The typical radiographic

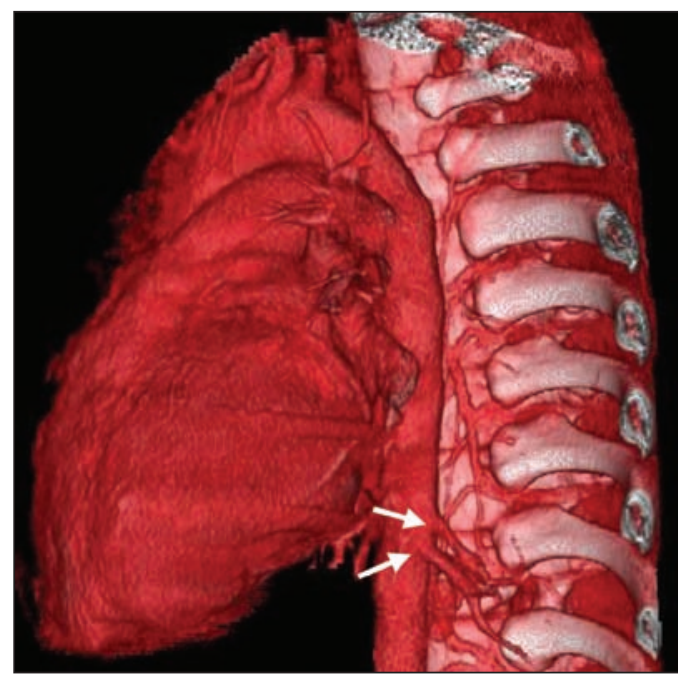

Figure 2: Reconstructed computed tomographic image showing two feeding arteries (arrows) from the aorta.

manifestation is focal opacification, especially on the left side. Rarely, a pulmonary sequestration appears as an area of hyper-radiolucency, as in our patient. This finding is called the "cystic form" of pulmonary sequestration, based on the radiographic appearance. Demonstration of systemic arterial supply, usually by contrastenhanced CT, is the critical diagnostic feature for differentiation from other pulmonary parenchymal abnormalities.

Recurrent infection can occur in intralobar sequestration, and surgical resection is supported by case series and expert opinion. ${ }^{2}$ For asymptomatic lesions, however, there is considerable debate about resection. The prognosis in pulmonary sequestration is variable and depends on the presence of accompanying malformations and perioperative complications.

\section{References}

1. Abbey P, Das CJ, Pangtey GS, et al. Imaging in bronchopulmonary sequestration. J Med Imaging Radiat Oncol 2009;53:22-31.

2. Corbett HJ, Humphrey GM. Pulmonary sequestration. Paediatr Respir Rev 2004;5:59-68.

3. Gezer S, Tastepe I, Sirmali M, et al. Pulmonary sequestration: a single-institutional series composed of 27 cases. J Thorac Cardiovasc Surg 2007;133:955-9. 